Meta

Journal des tradlucteurs

Translators' Journal

\title{
Traduction de textes destinés à des spécialistes : approche pédagogique
}

\section{Monique C. Cormier}

Volume 36, numéro 2-3, juin 1991

URI : https://id.erudit.org/iderudit/002394ar

DOI : https://doi.org/10.7202/002394ar

Aller au sommaire du numéro

Éditeur(s)

Les Presses de l'Université de Montréal

ISSN

0026-0452 (imprimé)

1492-1421 (numérique)

Découvrir la revue

Citer cet article

Cormier, M. C. (1991). Traduction de textes destinés à des spécialistes :

approche pédagogique. Meta, 36(2-3), 440-447. https://doi.org/10.7202/002394ar d'utilisation que vous pouvez consulter en ligne.

https://apropos.erudit.org/fr/usagers/politique-dutilisation/ 


\section{TRADUCTION DE TEXTES DESTINÉS À DES SPÉCIALISTES : APPROCHE PÉDAGOGIQUE}

MONIQUe C. CORMIER

Université de Montréal, Montréal, Canada

Toute méthode d'enseignement suppose une ventilation ou une décomposition des difficultés propres à la discipline en cause. En didactique de la traduction technique, une telle ventilation peut se faire à l'aide d'une typologie. La typologie retenue et qui distingue essentiellement trois types de textes, à savoir le texte de vulgarisation, le texte didactique et le texte destiné à des spécialistes (Cormier 1990a), permet d'accorder à l'activité de compréhension une progression ascendante.

La traduction de textes destinés à des spécialistes constitue la dernière étape à franchir dans un cours de traduction technique de premier niveau. Parce qu'il s'adresse à des spécialistes et que l'étudiant n'en est manifestement pas un, le texte spécialisé présente des difficultés de traduction plus grandes sur le plan de l'appréhension et de la compréhension que tout autre texte. Comme on ne peut traduire ce que l'on ne comprend pas, il faut bien sûr acquérir d'abord une certaine connaissance technique, c'est-à-dire une connaissance de compréhension et non nécessairement d'application, mais aussi être en mesure de faire des raisonnements techniques et de suivre une argumentation à caractère spécialisé. On verra une fois de plus que la terminologie n'est pas sans poser de problèmes, mais que la compréhension reste au cœur de la problématique.

La démarche pédagogique entreprise pour l'initiation au domaine traité est sensiblement la même, à quelques différences près, que celle que nous utilisons pour le texte didactique (Cormier 1990b).

$\mathrm{Au}$ lieu de distribuer un dossier technique aux étudiants, nous leur demandons de constituer eux-mêmes ce dossier en choisissant des textes en anglais et en français qui ont trait au domaine étudié.

Au moment où nous avons abordé le domaine des lave-autos, les étudiants disposaient d'une semaine pour constituer un dossier sur ce sujet. Même si la majorité d'entre eux avaient fait plusieurs fois l'expérience de faire laver une voiture dans ces postes de lavage, aucun n'était en mesure de faire la distinction entre un portique et un tunnel de lavage, ni d'expliquer le fonctionnement de tels postes. Il leur fallait donc constituer un dossier comportant des textes de vulgarisation ou des textes didactiques pour se familiariser avec les notions de base.

Dans un premier temps, les étudiants ont majoritairement dirigé leurs recherches vers les ouvrages encyclopédiques, monographies ou encore dictionnaires encyclopédiques. Comme on pouvait s'y attendre, en raison de l'existence relativement récente des postes de lavage, aucun des ouvrages consultés ne faisait même mention de telles installations.

Il fallait alors orienter les recherches vers d'autres sources documentaires, mais lesquelles? Nous ne voulions pas indiquer de nouvelles sources aux étudiants avant que ceux-ci n'aient réfléchi à la question. Cette recherche documentaire est en effet partie intégrante du cours au même titre que la traduction. Après tout, une fois sa formation terminée, l'apprenti traducteur devra se débrouiller seul. Au cours d'un échange avec les 
étudiants, l'un d'entre eux a fait remarquer que l'Office de la langue française avait publié un lexique des lave-autos et qu'une bibliographie y figurait. Comme ce lexique s'adressait autant aux spécialistes qu'au grand public, sa bibliographie contenait à la fois des documents vulgarisés et spécialisés. Les étudiants avaient donc eux-mêmes trouvé de nouvelles pistes de recherche et c'est à l'aide de cette bibliographie qu'ils ont constitué leur dossier technique.

À partir de l'ensemble des textes recueillis par les étudiants, la classe a fait un choix et retenu cinq documents, dont un document spécialisé en anglais d'une quarantaine de pages. $\grave{A}$ ces documents se sont ajoutées deux études terminologiques sur les termes «matériel» et «équipement».

À partir d'ici, la marche à suivre pour appréhender le nouveau domaine technique est identique à celle du texte didactique. On débute avec la documentation française. Une première lecture permet d'avoir une vue d'ensemble du domaine. Une seconde lecture permet de repérer la terminologie propre à ce domaine. Enfin, on passe à la compréhension et à l'organisation des notions. Encore une fois, le schéma explicatif a été utilisé et nous avons en outre demandé aux étudiants de faire comme exercice un résumé sur les différents types de postes de lavage automatique, leurs parties principales et leurs fonctions respectives. Le résumé et la contraction de textes constituent d'excellents moyens pour aider à dégager l'essentiel d'un domaine et à l'exprimer de façon concise. Quant à la documentation de langue anglaise, la même démarche a été suivie. Deux lectures, repérage de la terminologie et établissement d'une nomenclature bilingue.

Enfin, comme il n'y a rien de mieux pour se représenter un poste de lavage que d'en voir un, une visite à un lave-auto a été organisée et les étudiants ont pu profiter d'un échange avec l'un des opérateurs. Lorsqu'elles sont possibles, de telles visites sont profitables. Non seulement elles permettent aux étudiants de voir le matériel sur place et de poser des questions, mais elles les préparent à des démarches qu'ils auront souvent à entreprendre au cours de leur carrière. Les sources visuelles et orales constituent en effet des moyens de recherche documentaire aussi importants que les sources écrites et il faut savoir les utiliser.

Une fois que les étudiants ont circonscrit le domaine à l'étude, ils n'en sont pas pour autant devenus des spécialistes, mais ils sont prêts à appréhender la traduction des textes qui s'adressent à des spécialistes.

Le texte qui a fait l'objet de la traduction sur laquelle porte la présente analyse est extrait d'un document de la société américaine Exxon. Ce document, d'une quarantaine de pages, est un rapport sur les différents types de postes de lavage automatique. Au moment où ce texte a été rédigé, la société Exxon envisageait l'exploitation de postes de lavage et ce rapport avait pour but de présenter les conclusions d'un groupe de travail chargé d'étudier quel serait le type de poste de lavage susceptible d'être le plus avantageux, tant sur le plan financier qu'opérationnel. L'extrait retenu porte sur l'étude de l'équipement du tunnel de lavage et comprend certaines recommandations du groupe de travail.

\section{TUNNEL CAR WASH ${ }^{1}$ \\ EQUIPMENT INVESTIGATION}

In the study and review of many car wash sites and proposals, it soon became clear that there was little difference in the mechanical configuration of a car wash tunnel. It was the size and amount of equipment installed which varied in order to handle the anticipated capacities. Some exterior washes were tried with lighter and less expensive machinery in order to achieve a low cost installation. These were not generally found to be economically sound in the operation of the washing equipment. 
Several areas of mechanical configuration in the washing modules were proven to have very high operating and maintenance cost when expense records were checked. Generally these involved complex controls or actions which required repeated maintenance service in order to operate properly.

The tire cleaning equipment was found to have the greatest variance in standard of operation and operating economy. Exhibit $S$ illustrates the relative costs related to the steam-spin type wheel washer as compared with the horizontal brush wheel washing units. This differential in operating and investment costs along with the customer reaction problems when the spinner equipment contacts a vehicle's wheels supports the elimination of steam-spin type wheel washers from the tunnel equipment package. There is also a potential for damage and a safety hazard that has been associated with these units.

The top brush with knuckling action and reversing brush rotation, as included by some manufacturers, cannot be justified due to the excessive maintenance involved.

In development of a standardized package of equipment for nationwide uniformity and economy, it is recommended that hydraulically operated preplumbed prewired systems should be established. Though the validity of electric opposed to hydraulic operation of the system may yet be debated, the flexibility of hydraulic power has several advantages. The primary concern is meeting the Underwriters Label requirements. It has been proposed that the hydraulic system is economically advantageous when prewiring is specified.

Parmi tous les textes que les étudiants ont eu à traduire au cours de ce trimestre, ce texte est celui auquel ils ont opposé le plus de résistance, allant même jusqu'à demander de le retirer. Leur principale plainte concernait la difficulté de compréhension. À juste titre, ils n'osaient pas attaquer la traduction, se rendant compte qu'ils ne traduiraient que des suites de mots. C'est donc sur la compréhension que nous leur avons demandé de travailler. Ils ont formé des groupes de deux ou trois et se sont concentrés uniquement sur la compréhension, sans se préoccuper de la traduction. Chacun a pu se faire une idée de la signification du texte et la confronter avec celle de ses camarades. Puis, les étudiants ont entrepris leur propre traduction.

Compte tenu des difficultés du texte, les résultats ont été, dans l'ensemble, satisfaisants. Les erreurs relevées sont de deux types: imprécision lexicale et faux-sens commis à la suite de l'incompréhension de certains passages. Ces faux-sens sont dus à l'incompréhension de la langue de départ, à l'incompréhension de notions spécialisées, ou encore à l'inattention. Nous analyserons maintenant les principales erreurs commises, paragraphe par paragraphe. Pour éviter d'avoir à nous reporter au texte anglais, nous reprendrons au besoin certains passages. Chaque fois qu'une partie de l'interprétation d'un étudiant sera en italique, elle le sera de notre fait.

La fin de la première phrase du texte a été l'objet, notamment, de deux faux-sens que nous nous expliquons mal, sinon qu'ils sont le résultat d'une inattention.

«[...] it soon became clear that there was little difference in the mechanical configuration of a car wash tunnel.»

La traduction de ce passage a donné ce qui suit chez deux étudiants :

«[...] il devint vite évident que peu de différence existait dans les façons de conception mécanique d'un poste de lavage.»

«Les études réalisées à partir de plans d'aménagement et de la disposition mécanique de postes de lavage démontrent peu de différences avec le tunnel de lavage.»

Dans la première interprétation, l'étudiant a utilisé pour traduire l'unité car wash tunnel le générique «poste de lavage» au lieu du spécifique «tunnel de lavage». Comme le poste de lavage est constitué soit d'un tunnel de lavage, soit d'un portique de lavage, 
l'utilisation de «poste de lavage» entraîne ici un faux-sens. Ces notions se présentent en effet comme suit :

\section{Figure I}

Relation générique - spécifique : poste de lavage automatique

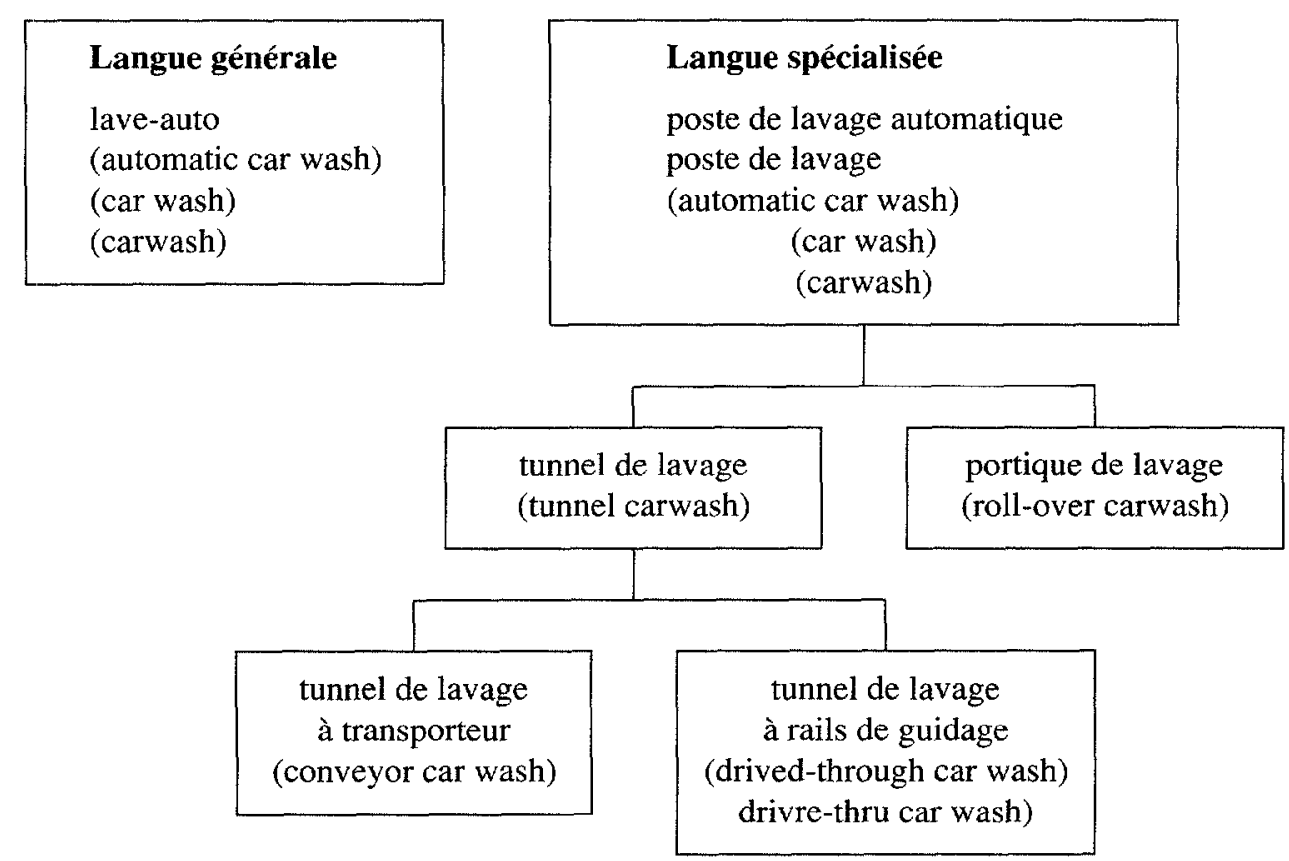

Dans certains contextes, s'il est parfois possible de rendre un spécifique par un générique sans pour autant commettre un faux-sens, il était ici impossible de se servir du générique sans que le sens du passage en soit modifié. Par ailleurs, en plus de l'utilisation du passé simple qui ne convient pas du tout dans le texte technique (on aurait pu se servir du présent de l'indicatif), on remarque la traduction maladroite du terme anglais configuration, sans doute dictée par la crainte des faux-amis. Pourtant, le terme français «configuration» convenait très bien.

Dans la seconde interprétation, l'étudiant a pensé que la configuration mécanique d'un poste de lavage était semblable à celle d'un tunnel de lavage, ce qui est tout à fait illogique lorsqu'on prend la peine d'y réfléchir. De fait, le passage anglais voulait simplement dire que la configuration mécanique varie peu d'un tunnel de lavage à un autre. Enfin, le terme «disposition» n'était pas mauvais, mais l'étudiant aurait pu conserver le terme «configuration» utilisé dans plusieurs domaines techniques.

Une deuxième erreur mérite d'être soulignée dans le premier paragraphe :

«Some exterior washes were tried with lighter and less expensive machinery in order to achieve a low cost installation. These were not generally found to be economically sound in the operation of the washing equipment.» 
L'unité washing equipment de la fin du paragraphe a été mal comprise par près du tiers des étudiants. Le texte anglais signifie que l'utilisation d'une machinerie moins lourde et moins coûteuse pour les lavages extérieurs se révèle non rentable, surtout en ce qui a trait à l'«unité de lavage». Le terme washing equipment est ici synonyme de washing unit et a pour correspondant «unité de lavage». Comme le montrent les interprétations qui suivent, on a confondu l'unité de lavage avec le poste de lavage, la première faisant partie du second:

«Dans le but de réaliser des économies à l'installation, des postes de lavage ont été dotés d'équipements moins lourds et moins coûteux. En général, cette tentative n'a pas permis de rentabiliser l'exploitation des postes de lavage.»

«Des postes de lavage comprenant une machinerie moins lourde et moins coûteuse ont été mis à l'essai de façon à diminuer les coûts d'installation. En général, l'exploitation de tels postes de lavage ne s'est pas avérée rentable.»

Un tunnel de lavage comprend généralement une rampe de prélavage, une unité de lavage, une rampe de rinçage et de cirage et enfin, une soufflerie. Les interprétations de ces étudiants comportent une part de vérité. En effet, si l'unité de lavage n'est pas rentable, le poste de lavage dans son ensemble ne l'est donc pas. Mais en associant l'unité anglaise washing equipment au correspondant français «poste de lavage», ils ont omis de préciser que l'utilisation de cette machinerie était peu rentable, particulièrement en ce qui a trait à l'unité de lavage. Ce ne sont donc pas toutes les installations du poste de lavage qui sont à l'origine de la non-rentabilité des postes, mais uniquement l'unité de lavage. En traduisant comme ils l'ont fait, les étudiants ont donc privé les destinataires éventuels de la traduction d'une information importante.

$\mathrm{Au}$ second paragraphe, la première phrase a été l'objet d'un faux-sens et d'une imprécision lexicale alors que la deuxième phrase de ce même paragraphe a donné lieu à un faux-sens.

«Several areas of mechanical configuration in the washing modules were proven to have very high operating and maintenance cost when expense records were checked. Generally these involved complex controls or actions which required repeated maintenance service in order to operate properly.»

Une fois de plus, un étudiant sur trois a associé l'unité washing modules aux postes de lavage alors qu'il s'agissait d'unités de lavage.

«Une vérification des comptes de frais montre que plusieurs unités du poste de lavage ont des frais d'exploitation et d'entretien ćlevés. Dans l'ensemble, leurs commandes et mécanismes compliqués nécessitent un entretien répété pour bien fonctionner.»

«Il a été prouvé par l'étude des bilans de dépenses que plusieurs parties mécaniques des laves-autos occasionnaient un coût opérationnel et des frais d'entretien très élevés. Il fallait, pour assurer leur bon fonctionnement, procéder à des manceuvres ou à des contrôles très compliqués qui nécessitaient un entretien soutenu.»

On voit par les interprétations qui précèdent que les étudiants ne se sont pas arrêtés à la signification du terme anglais modules qui désigne «unités d'un ensemble». Dès qu'ils ont aperçu le mot washing associé à un autre mot, plusieurs étudiants y ont fait correspondre le terme «poste de lavage». Le contexte n'est pourtant pas ambigu: ce paragraphe ne fait qu'expliciter ce qui précède au premier paragraphe. Et pourtant, certains de ceux qui ont fait ce faux-sens avaient bien saisi le sens du premier paragraphe.

Toujours dans cette même phrase, neuf étudiants sur dix ont vu dans expense (expense records) la signification accordée à ce mot dans la langue générale et l'ont donc 
traduit par «dépenses». Dans le domaine de la comptabilité, ce terme a rarement comme correspondant français «dépenses», mais plutôt «frais» ou «charges». Quant au terme «dépenses» utilisé dans le domaine de la comptabilité, il a généralement comme correspondant anglais le terme expenditures. Puisque les charges comprennent à la fois les achats et les frais, et qu'il n'est pas question d'achats dans le contexte, mais bien de coûts d'exploitation et d'entretien, le terme «frais» est ici le correspondant de expense. Les expense records sont donc des relevés de frais.

Il est intéressant de remarquer les effets que peut avoir l'interférence linguistique sur la traduction. Dans la seconde phrase du deuxième paragraphe, l'anglais dit:

«Generally these involved complex controls or actions which required repeated maintenance service in order to operate properly.»

L'unité controls or actions a été tout de suite assimilée par certains étudiants à des contrôles et des actions, et l'on a alors retenu l'aspect «actif» de ces deux éléments. Plusieurs d'entre eux ont ainsi compris qu'il fallait procéder à des manœuvres et à des contrôles compliqués pour s'assurer du bon fonctionnement des unités de lavage. Or les controls or actions sont de fait les commandes et les mécanismes des unités de lavage. On aurait alors pu traduire cette phrase de la façon suivante:

«En général, ces installations sont dotées de commandes ou de mécanismes compliqués qui ne peuvent fonctionner convenablement sans le concours d'un service d'entretien régulier.»

Le manque de connaissances thématiques peut aussi être à l'origine d'erreurs de traduction. En effet, l'étudiant n'est pas toujours au courant des réalités de la vie politique, économique et sociale et, la méconnaissance de telles réalités est souvent préjudiciable à la traduction. La traduction d'un passage du dernier paragraphe l'illustre bien. Rappelons d'abord le passage anglais:

«Though the validity of electric opposed to hydraulic power operation of the system may yet be debated, the flexibility of hydraulic power has several advantages. The primary concern is meeting the Underwriters Label.»

La dernière phrase de ce passage a été mal comprise par la majorité des étudiants. Comme ils n'ont pas saisi la signification de Underwriters Label, le sens de la phrase entière est resté impénétrable. Les interprétations suivantes en témoignent:

«Le souci premier est de respecter les exigences du syndicat de garantie désigné.»

«Il est primordial de satisfaire aux normes relatives à la marque de garantie.»

«Il s'agit premièrement de satisfaire aux normes et spécifications du pays.»

"L'objectif premier est toutefois de satisfaire aux exigences du label.»

Il ne s'agit pas ici de relever des perles, certaines de ces traductions provenant d'ailleurs de bons étudiants, mais de montrer les conséquences de l'incompréhension sur la traduction. C'est sans doute l'expression Underwriters Label qui aura mis les étudiants sur une fausse piste et, il faut le dire, l'arrivée un peu brutale de cette phrase dans le contexte. Mais on doit se rappeler que ce rapport s'adresse à des spécialistes et que l'auteur va directement au but, présumant que les destinataires auront les connaissances nécessaires pour comprendre le texte.

Si l'on revient au passage anglais, on comprend que l'utilisation de l'énergie hydraulique plutôt que l'énergie électrique comporte plusieurs avantages, notamment celui de répondre aux exigences du Underwriters Label. En plus de ne pas avoir compris ce que cette phrase venait faire dans le texte, les étudiants ne savaient pas ce qu'était le 
Underwriters Label. L'utilisation de majuscules aurait dû les mettre sur une piste: s'agissait-il d'un organisme officiel, d'une entreprise, etc.? Pourtant c'est à l'aide de dictionnaires qu'ils ont tenté de résoudre le problème avec les résultats que l'on sait.

Le Underwriters Label, qui est un organisme américain, porte aujourd'hui le nom de Underwriters Laboratories et a pour homologue canadien le Underwriters Laboratories of Canada. Ces laboratoires d'assureurs sont généralement des organismes à but non lucratif qui exploitent des laboratoires et un service d'homologation. Divers dispositifs, accessoires, matériaux de construction et services y sont examinés et mis à l'essai dans le but de vérifier leur résistance au feu ou d'en déterminer les risques.

Avec de tels renseignements, qu'une simple consultation téléphonique aurait pu fournir aux étudiants, la phrase dévoile tout son sens. On se rend ainsi compte que l'utilisation de l'énergie hydraulique est conforme aux normes du Underwriters Label (devenu le Underwriters Laboratories).

En relisant les interprétations des étudiants, une chose nous frappe. Leur traduction est incompréhensible parce qu'aucun d'entre eux n'a réellement saisi la signification du Underwriters Label. La connaissance thématique a fait défaut, empêchant toute pénétration du texte et, par conséquent, toute formulation intelligible en français.

Malgré le caractère ponctuel des erreurs relevées dans la traduction du texte destiné à des spécialistes, ces erreurs permettent de faire l'observation suivante: le problème principal auquel l'étudiant fait face dans l'apprentissage de la traduction technique de premier niveau est la compréhension, élément pivot qui ouvre la voie au message, puis à sa réexpression. Sans compréhension, le texte reste hermétique, ce qui empêche qu'un sens puisse s'en dégager et qu'une réexpression intelligible puisse s'opérer.

Quelles sont les causes de l'incompréhension, si fréquente dans l'apprentissage de la traduction? L'incompréhension est le résultat d'une faille dans la connaissance linguistique, dans la connaissance thématique, ou dans les deux.

Lorsque l'incompréhension est liée à la connaissance linguistique, c'est soit la connaissance de la langue de départ qui n'est pas suffisamment complète pour permettre la compréhension, soit l'interférence entre l'anglais et le français qui vient s'immiscer dans la compréhension et la brouiller, soit encore l'interférence entre la langue générale et la langue de spécialité qui la fait dévier. Le problème de l'interférence est, de tous, le plus sournois parce que, en associant une signification à des mots, l'étudiant croit comprendre le message alors qu'en fait, il s'engage dans une voie sans issue.

La méconnaissance d'une partie ou de la totalité du sujet traité dans un texte à traduire peut aussi entraver la compréhension et faire en sorte que l'étudiant traduise pour comprendre au lieu de comprendre pour traduire. En outre, comme le révèle l'analyse des erreurs, l'incompréhension peut être le résultat de l'incapacité de rattacher une information nouvelle à des connaissances antérieures, phénomène que l'on a trop tendance à ignorer, mais que Danica Seleskovitch (1968:93) a observé :

«[...] s'il y a identité entre l'information reçue et la connaissance antérieure, le rattachement est direct et la compréhension équivaut à une re-connaissance ${ }^{2}$. S'il y a un trop grand écart entre l'information et les connaissances antérieures, le rattachement n'est pas possible, et il y a incompréhension. [...] La compréhension rattache l'information à des connaissances voisines; si celles-ci sont absentes, l'information passe inaperçue.»

Pour prévenir le plus possible ces erreurs, l'enseignant doit prévoir dans sa démarche pédagogique un certain nombre d'étapes exposées précédemment et qui visent une préparation approfondie des sujets traités, tant sur le plan de la documentation que sur celui de la terminologie. En outre, il est impératif que les techniques de recherche documentaire et terminologique soient enseignées et mises en application au même titre que l'est la méthode de traduction. 


\section{NOTES}

1. Exxon Company (1973): Project Report on Car Wash Equipment Evaluation, Unpublished Report, Exxon Company, U.S.A.

2. Souligné dans le texte.

\section{BIBLIOGRAPHIE}

CORMIER, Monique C. (1990a): «Proposition d'une typologie pour l'enseignement de la traduction technique», Marianne Lederer (dir.), Les Etudes traductologiques, Paris, Minard Lettres Modernes.

CORMIER, Monique C. (1990b) : «Traduction de textes de vulgarisation et de textes didactiques : approche pédagogique", Meta, 35-4, pp. 676-688

SELESKOVITCH, Danica (1968): L'interprète dans les conférences internationales, Paris, Minard Lettres Modernes. 